\title{
From Linear Representations to Object Parts
}

\author{
Gabriella Sanniti di Baja, L. Serino, and Carlo Arcelli \\ Institute of Cybernetics "E.Caianiello", CNR \\ Via Campi Flegrei 34, 80078 Pozzuoli, Naples, Italy \\ \{g.sannitidibaja, 1.serino,c.arcelli\}@cib.na.cnr.it
}

\begin{abstract}
The use of the skeleton for object decomposition in the framework of the structural approach to shape description is discussed. Object decomposition is obtained starting from a suitable partition of the skeleton. The elements of the skeleton partition are then used as seeds from which to recover the various regions into which the object is decomposed. A merging process is also accomplished so as to have a final decomposition in accordance with human perception and stable when the object is available in different poses or sizes.
\end{abstract}

\section{Introduction}

The description of a digital object with complex shape can be facilitated by following the structural approach in analogy with the performance of human observers. According to this approach, the object is decomposed into a number of parts characterized by simple shape. Then, the description of the object is given in terms of the description of the parts into which the object has been decomposed as well as in terms of the spatial relationships among the parts.

Shape understanding has been investigated both in the literature on perceptual psychology and by computer scientists willing to introduce tools allowing machines to understand shape in the same way human beings do [1-3].

Shape parsing into components can be obtained by working on an object's representation scheme, such as boundary representation and region-based representation [4-7]. Boundary representation has been used mainly for 2D object decomposition, where the minima rule and the short-cut rule have been proposed and used successfully. The extension of these rules to the case of $3 \mathrm{D}$ objects is rather complex and only a limited number of methods have been suggested. In turn, regionbased representation, namely the skeleton, has been used in case of both $2 \mathrm{D}$ and $3 \mathrm{D}$ objects, where object's decomposition is achieved via skeleton partition. Decomposition guided by skeleton partition has been followed particularly for objects perceived as composed by ribbon-like parts. In fact, the skeleton of an object is a subset of the object, with the same topology as the object and consisting of curves symmetrically placed within the object. Thus, in case of a ribbon-like object, a one-toone correspondence is immediately clear between the curves composing the skeleton and the parts composing the object. Actually, also in case of objects with variable thickness and shape, the skeleton can still be used to guide object's decomposition, 
but a suitable grouping of some of the curves composing the skeleton is necessary to establish a correct correspondence between skeleton subsets and object parts.

Most of the skeletonization methods have been influenced by the work of Blum on medial axis transform MAT [8]. The MAT is defined in the Euclidean space as the locus of the symmetry points, i.e., the points of the object that can be seen as centers of balls tangent to the object boundary in at least two distinct parts and included in the object. Each MAT point is associated the radius of the corresponding ball. The object can be recovered by the envelope of the balls centered on the symmetry points.

For any 2D object and for 3D ribbon-like objects, symmetry points are placed along the symmetry axes of the object. Thus, the skeleton coincides with the MAT and full object recovery is possible starting from the skeleton. In turn, for 3D objects with variable thickness and shape, symmetry points are placed along symmetry planes and symmetry axes so that the MAT consists of surfaces (and curves) symmetrically placed within the object. For objects that do not include cavities, a linear subset of the MAT can still be extracted so as to obtain the skeleton, but full object's recovery starting from the skeleton cannot be guaranteed, since obviously the skeleton cannot include all symmetry points of the object.

The computation of the medial axis transform is a heavy job. Thus, a discrete approximation of the MAT based on the detection of the centers of maximal balls in the distance transform of the object is of interest. In the distance transform, object's voxels are labeled with their distance from the complement of the object. In this way, each voxel can be interpreted as the center of a ball with radius equal to the distance value of the voxel itself. Any ball that is not included by any other single ball in the object is called maximal ball and its center is called center of maximal ball. Centers of maximal balls are obviously symmetry points. Their detection can be easily accomplished by comparing the distance values of neighboring voxels. Moreover, any ball can be built by applying the reverse distance transformation [9] to its center and the object can be recovered by applying the reverse distance transformation to its centers of maximal balls. Thus, the MAT can be approximated by the set of centers of maximal balls. Unfortunately, such a set is not guaranteed to be connected even if the object is connected, so that skeletonization algorithms should identify as skeletal voxels also other voxels in the distance transform with the purpose of obtaining a representation scheme topologically equivalent to the object. Moreover, while the only centers of maximal balls are not enough to guarantee topological correctness of the skeleton, many of them have to be necessarily disregarded to guarantee linearity of the representation scheme in the presence of 3D objects that are not ribbon-like. Thus, skeletonization algorithms should select among all centers of maximal balls the most important ones to preserve shape information as much as possible.

In this paper, we will describe an object's decomposition method based on a suitable partition of the skeleton. We will use the skeletonization algorithm described in [10], which originates a topologically correct skeleton, symmetrically placed within the object and representing the object's shape sufficiently well even if -as explained before- only partial object recovery is possible starting from it. The skeleton will be partitioned into three types of subsets, called simple curves, complex sets, and single points. The partition components will then be used as seeds to reconstruct the associated object's parts, which will be respectively called simple regions, kernels, and bumps. Kernels are interpreted as sort of main bodies from which simple regions 
and bumps protrude. Any simple region will be furthermore divided into basic regions, by taking into account the variation in curvature and distance value along the corresponding simple curve, which will be divided into segments by means of polygonal approximation. A merging process will also be taken into account so that the final object decomposition results to be in accordance with human perception.

\section{Notions and Definitions}

We deal with binary 3D images in cubic grids and consider objects without cavities. To avoid topological paradoxes, we use the 26-connectedness for the object and the 6connectedness for the background.

The $3 \times 3 \times 3$ neighborhood $\mathrm{N}(p)$ of any object voxel $p$ is the set including the six face-, the twelve edge- and the eight vertex-neighbors of $p$.

The path-based distance between two voxels $p$ and $q$ is measured by the length of a minimal discrete path linking $p$ to $q$. In order such a length is a good approximation of the Euclidean length of the path, we use the weights $w_{f}=3, w_{e}=4$ and $w_{v}=5$, as suggested in [9], to measure the unit moves from any voxel towards its face-, edgeand vertex-neighbors along the path, respectively. The obtained distance function is referred to as the $<3,4,5\rangle$ weighted distance and provides a good approximation to the Euclidean distance. In fact, as shown in [11], if the minimization of the absolute difference from the Euclidean distance in a $\mathrm{M} \times \mathrm{M} \times \mathrm{M}$ image is used as evaluation criterion, the maximal error with the $<3,4,5>$ weighted distance is $0.1181 \mathrm{M}$.

The $<3,4,5>$ distance transform DT of the object is a replica of the object, where voxels are labeled with the length of a shortest path to the background. The $k$-th layer of DT is the set of voxels with distance value $d$ such that $3 \times(k-1)<d \leq 3 \times \mathrm{k}[12]$.

The skeleton of an object is a subset of the object with linear structure, topologically equivalent to the object and symmetrically placed within the object. In this paper we refer to the skeletonization method [10] based on the $<3,4,5>$ distance transform. Each skeleton voxel is labeled with its $<3,4,5>$ weighted distance from the complement of the input object. Due to the good approximation of the Euclidean distance provided by the $<3,4,5>$ weighted distance, such a skeleton is stable under isometric transformations of the input. A voxel $p$ of the skeleton is an end point when it has only one neighboring skeleton voxel in $\mathrm{N}(p)$. A voxel $p$ of the skeleton is a branch point when it has more than two neighboring skeleton voxels in $\mathrm{N}(p)$.

The ball associated to any skeleton voxel $p$ is obtained by applying to $p$ the reverse distance transformation. When the reverse distance transformation is applied to skeleton voxels that are neighbors of each other or constitute pairs of voxels closer to each other than the sum of the corresponding radii, the obtained balls partially overlap with each other and form a unique connected component. Such a connected component is called zone of influence of the skeleton voxels to which the reverse distance transformation has been applied. The zones of influence obtained by applying the reverse distance transformation to the branch points will be used in this paper to obtain the partition of the skeleton.

The polygonal approximation of an open simple curve can be obtained by using the split type algorithm suggested by Ramer [13]. The two extremes of the open curve are taken as vertices of the polygonal approximation. To identify the other vertices, we 
consider the straight line joining the two extremes of the curve and compute the Euclidean distance of all points of the curve from such a straight line; we take as vertex the point with the largest distance, provided that such a distance is greater than an a priori fixed threshold (to be set depending on the desired approximation quality). Each found vertex divides the curve into two curves, to each of which the splitting procedure is applied. The process continues as far as new vertices are detected.

Local concavity filling can be used to make planar the surface separating two adjacent regions. To this purpose, voxels belonging to one of the two regions, say to region $\mathrm{H}$, and placed in local concavities of the adjacent region, say region $\mathrm{K}$, are removed from $\mathrm{H}$ and are assigned to $\mathrm{K}$. This process is iterated until no more local concavities can be found along the surface separating $\mathrm{H}$ from $\mathrm{K}$. The concavity filling algorithm used in this paper is based on the use of $3 \times 3 \times 3$ operations [14].

\section{Skeleton Partition}

To partition the skeleton into the three desired types of subsets (simple curves, complex sets, and single points) we identify the branch points in the skeleton and use their associated zones of influence.

We point out that often, more than one single branch point is found in the skeleton in correspondence with configurations where a human observer would expect to find just one branch point. However, in any such a case the branch points are likely to be very close to each other, so that the balls associated to these branch points partially overlap and a single zone of influence is generated from them. In other words, the number of zones of influence is likely to be smaller than the number of branch points, and a single zone of influence is obtained in correspondence with each configuration where a single branch point would characterize the ideal skeleton.

Each connected component of skeletal voxels that are not included in any zone of influence constitutes a simple curve of the partition. End points of the skeleton that are included in zones of influence constitute single points of the partition. When single points exist, the skeleton branches that can be traced from the corresponding end points until branch points are met are called linking branches. Each connected component of skeletal voxels included in a zone of influence, except for end points and associated linking branches possibly found in that zone of influence, constitutes a complex set of the partition. We note that single points are not very frequent.

Fig.1 shows two objects and the partition into simple curves, single points and complex sets for their skeletons. The partition of the skeleton of the first object, "camel", includes four complex sets (red), thirteen simple curves (green) and one single point (pale blue) with an associated linking branch (gray), while the partition of the skeleton of the second object, "dog", includes only three complex sets and ten simple curves. We note that single points are not very frequently found in the partition of the skeleton.

Simple curves are furthermore divided into segments by means of polygonal approximation. Since voxels of a simple curve are labeled with their distance from the complement of the object, both changes in geometry along the simple curve at hand and changes in distance value of its voxels will be used to identify the segments into 
which the simple curve will be divided. Curvature and distance value of skeletal voxels are related to the shape of the region associated with the simple curve by the reverse distance transformation. For such a region, changes in curvature and distance value along the corresponding simple curve respectively reflect curvature variations along the surface separating the region from the complement of the object, and thickness variations of the region itself.
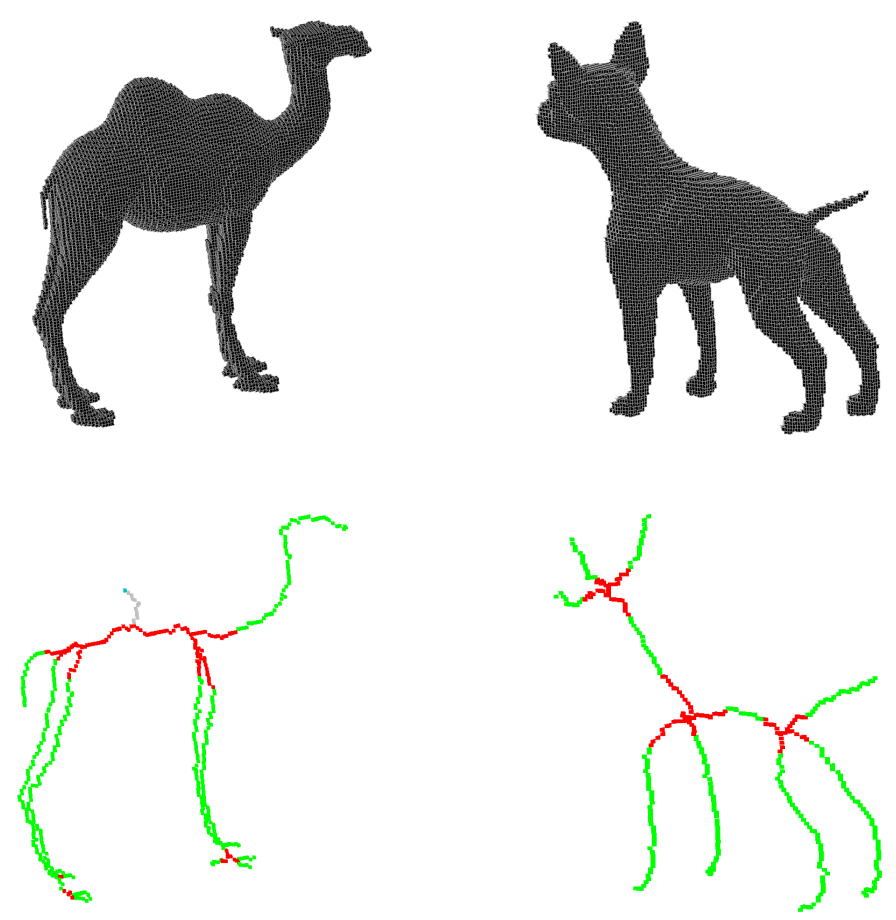

Fig. 1. The objects "camel" and "dog", top, and the partition of their skeletons into simple curves, green, single points, pale blue, and complex sets, red, bottom. The linking branch, connecting the single point existing in the skeleton of "camel" to the complex set is shown in gray.

To take into account simultaneously both changes in geometry along the simple curve at hand and changes in distance value of its voxels, we should represent the curve in a 4D discrete space, where the coordinates are the three Cartesian coordinates and the distance value of the voxels of the $3 \mathrm{D}$ simple curve. We note that in the $3 \mathrm{D}$ space, each simple curve found in the partition of the skeleton is a connected set. Each voxel of the simple curve has exactly two neighbors in the curve, with the exception of the two extremes of the curve having only one neighbor in the curve. However, when we represent a $3 \mathrm{D}$ simple curve in the above 4D space, connectedness is not guaranteed and voxels of the curve may result as a sparse set of points in the 4D space. On the other hand, we note that the skeleton [10] has been computed by using the $<3,4,5>$ distance transform, where the layers can be easily 
detected. Moreover, we note that adjacent skeleton voxels either belong to the same layer or to layers whose indexes differ by one unit. Thus, by using as the $4^{\text {th }}$ coordinate of each point in the 4D space the index of the layer to which the corresponding curve voxel belongs in place of the distance value, connectedness is maintained when passing from the $3 \mathrm{D}$ to the $4 \mathrm{D}$ representation.

The square of the Euclidean distance $d$ of a point $\mathrm{C}$ from a straight line $\mathrm{AB}$ passing through the two extremes of a simple curve is computed as:

$$
d^{2}=\|\mathrm{AC}\|^{2}-\mathrm{P}_{\mathrm{ABC}} * \mathrm{P}_{\mathrm{ABC}} /\|\mathrm{AB}\|^{2}
$$

where $\|\mathrm{AB}\|$ denotes the norm of the vector $\mathrm{AB}$, and $\mathrm{P}_{\mathrm{ABC}}$ the scalar product between vectors $\mathrm{AB}$ and $\mathrm{AC}$.

We have experimentally found that a threshold $\gamma=20$ for the distance is generally adequate to originate a polygonal approximation sufficiently faithful to the original simple curve and at the same time able to prevent an excessive fragmentation of the simple curve.

Once the polygonal approximation has been accomplished on all simple curves and all vertices have been detected in the $4 \mathrm{D}$ space, we go back to the representation of the skeleton in the 3D space and mark there the skeleton voxels corresponding to the found vertices. See Fig. 2, where the vertices identified on the simple curves of the skeleton of the objects in Fig. 1 are shown as black voxels. Consecutive segments share a common vertex, that we call hinge.
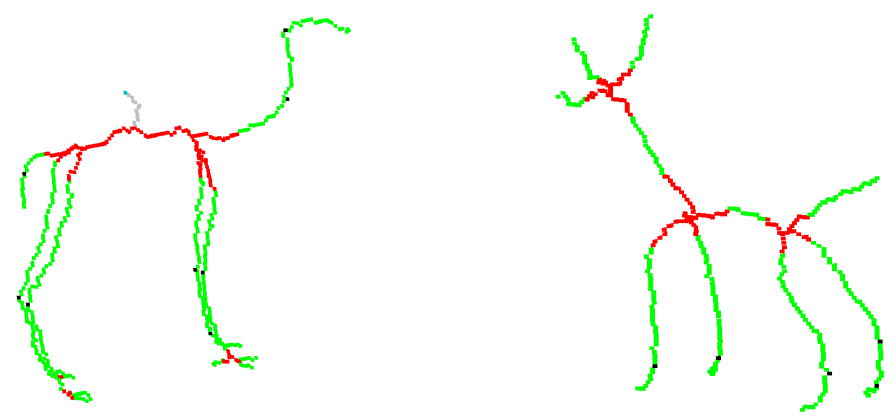

Fig. 2. Black voxels denote vertices found when performing polygonal approximation of the simple curves in the partition of the skeleton

\section{Object Decomposition}

A process in two steps is accomplished to build the kernels, bumps and simple regions into which the object is decomposed and to divide the simple regions into a number of basic regions. The first step is based on the reverse distance transformation applied to the individual skeleton partition components. The second step is done to achieve the complete recovery of the various regions. In fact, the skeleton of a 3D object does not generally include all centers of maximal balls. Thus, during the second step, object 
voxels that have not been recovered by the reverse distance transformation during the first step, are assigned to the region to which they result to be closer. Preliminarily, connected component labeling is accomplished to assign an identity label to all the seeds of the partition, i.e., the complex sets, single points, simple curves, segments and hinges. Actually, a unique identity label is assigned to all hinges.

During the first step, reverse distance transformation with propagation of the identity label is accomplished in two distinct arrays. In the first array, say A1, the process is applied to each complex set, single point and simple curve, while in the second array, say A2, the process is applied to segments and hinges. In any case, care is taken to properly assign the identity label to object voxels that can be recovered by more than one partition seed. In A1, voxels recovered by more than one seed are assigned the identity label of the closest complex set. In A2, voxels recovered by more than one seed are assigned the unique identity label characterizing the hinges.
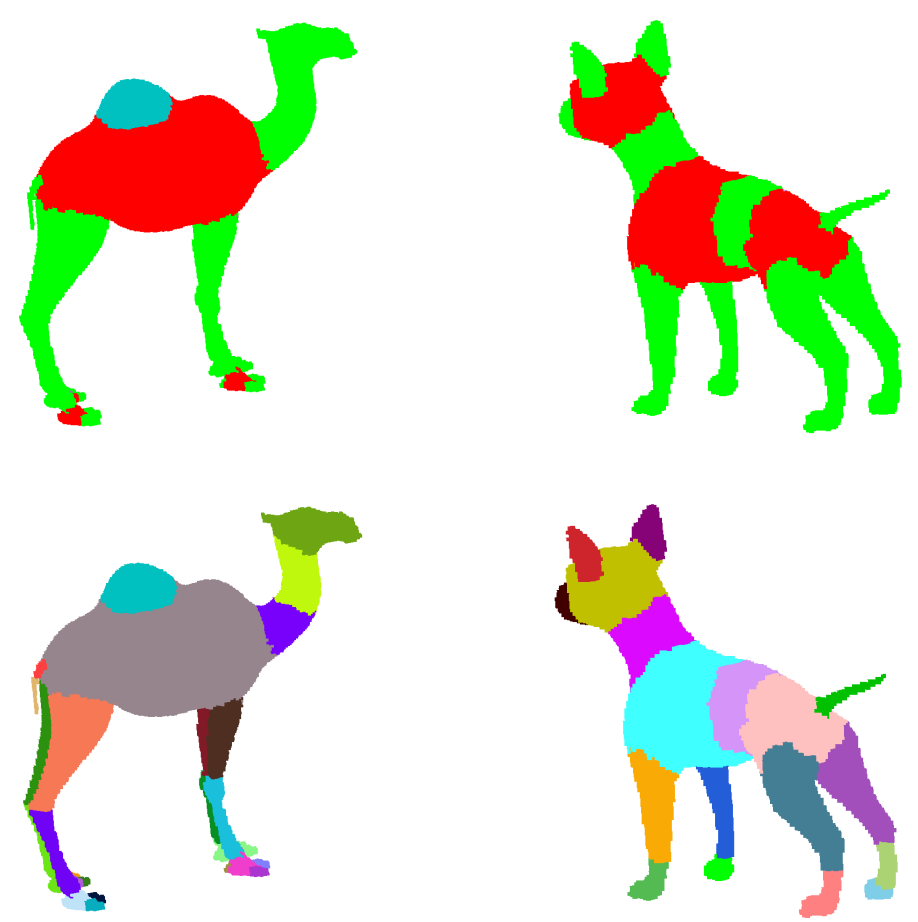

Fig. 3. Top, decomposition into kernels (red), bumps (pale blue) and simple regions (green). Bottom, decomposition where each region has the identity label of the corresponding seed and simple regions are divided into basic regions.

Before completing object recovery, a process is done in A1 to make planar the surfaces separating the regions obtained so far. To this aim, concavity filling is used to change the identity labels of voxels labeled as voxels recovered by complex sets and that are placed in local concavities of regions recovered from simple curves or 
single points. The process is iterated until almost planar surfaces separate regions associated to simple curves or single points from regions associated to complex sets.

The second step is then accomplished in both arrays A1 and A2 by assigning to the object voxels that have not been recovered during the first step and to the voxels with the unique identity label assigned to the hinges, the identity label of the region to which they are closer. The decomposition obtained in A1 is into kernels, bumps and simple regions. By using the results present in $\mathrm{A} 1$ and $\mathrm{A} 2$, the decomposition where simple regions are furthermore divided into basic regions is obtained.

As an example, in Fig. 3 top the decomposition into kernels, bumps and simple regions is shown, while the decomposition where the simple regions are furthermore divided into basic regions is given in Fig. 3 bottom.

\section{$5 \quad$ Region Merging}

The obtained decomposition into kernels, simple regions (possibly divided into basic regions) and bumps satisfies the one-to-one correspondence between such object parts and the complex sets, simple curves (possibly divided into segments) and single points of the partition of the skeleton. However, the number of parts may be larger than the number of regions perceived as individually significant by an observer. Thus, merging can be done to reduce the number of object parts and obtain a decomposition that is more stable when the same object is available in different poses or sizes.

Since kernels can be interpreted as main bodies of the object, we candidate bumps and simple regions to be merged to the kernels from which they protrude. In particular, if a simple region is divided into basic regions, we candidate to merging the whole simple region. Bumps are always adjacent to one kernel only, while simple regions may be adjacent to one kernel only (and in this case we call them peripheral regions), or can be delimited by two kernels (and in this case we call them nonperipheral regions). Only regions with small relevance will be merged.

To evaluate the relevance of a region we use criteria based on visibility and on volume. The surface of a region $\mathrm{R}$ is the set of voxels of $\mathrm{R}$ with a face-neighbor in either the background or in (any of) the delimiting kernel(s). The surface is interpreted as consisting of a visible portion, including the voxels with a faceneighbor in the background, and a non-visible portion, including the voxels with a face neighbor in the adjacent kernel(s). By respectively denoting the area of the visible and the non-visible portions as $A_{v}$ and $A_{n v}, R$ is regarded as scarcely visible, and hence can be merged, if the ratio $A_{v} / A_{n v}$ is smaller than an a priori fixed threshold $\theta$. As far as volume is concerned, let $\mathrm{R}$ be region adjacent to a kernel $\mathrm{K}$. Merging of $\mathrm{R}$ to $\mathrm{K}$ can be accomplished if the ratio between the volume of the region union of $\mathrm{R}$ and $\mathrm{K}$, and the volume of $\mathrm{K}$ is smaller than an a priori fixed threshold $\tau$.

The first merging step is concerned with non-peripheral simple regions and involves only the visibility criterion. To decide whether any such a region should be merged into a unique object part together with the two delimiting kernels, we set $\theta=2$. This setting is done by considering a continuous cylinder with height $h$, for which it is $\mathrm{A}_{\mathrm{v}} / \mathrm{A}_{\mathrm{nv}}=2 \pi r h / 2 \pi r^{2}$, as scarcely visible if $h$ is smaller than the diameter $2 r$. 
Actually, a kernel $\mathrm{K}$ may be adjacent to more than one non-peripheral simple region. Thus, if the visibility criterion is satisfied for all the simple regions adjacent to $\mathrm{K}$, an excessive merging may originate. To avoid such a drawback, we associate a multiplicity degree $m$ to each kernel, by counting the number of adjacent nonperipheral simple regions satisfying the visibility criterion, and perform merging by analyzing regions in increasing order of multiplicity degree.

If a non-peripheral simple region $S_{j}$ satisfying the visibility criterion is delimited by kernels having both $m=1$, the three regions are merged into a unique region, whose identity label will be the identity label of the kernel with the largest volume.

If for $S_{\mathrm{j}}$ one of the two delimiting kernels has $m=1$, while the second kernel has $m>1$, we first merge $S_{\mathrm{j}}$ to the kernel characterized by $m=1$, and the resulting merged region is ascribed the identity label of that kernel.

Once merging involving all kernels with $m=1$ has been accomplished, kernels with $m>1$ are considered. If for the inspected kernel $\mathrm{K}_{\mathrm{i}}$ the $m$ adjacent regions have already been merged to other kernels, $K_{i}$ is assigned to the adjacent region with which it shares the largest portion of its surface, among the $m$ possible ones.

The second merging step is concerned with peripheral regions, i.e., bumps and simple regions. The visibility criterion is integrated by the condition on volume. Using the visibility criterion is done to avoid merging elongated and narrow regions that satisfy the condition on volume. According to our model, where it is $h<2 r$, the default value for the threshold results $\theta=5$. In fact, we regard merging of a cylindrical peripheral region $\mathrm{R}$ as possibile if $\mathrm{A}_{\mathrm{v}} / \mathrm{A}_{\mathrm{nv}}=2 \pi r h / \pi r^{2}<\theta$. As for the value of $\tau$, we have experimentally found that satisfactory results are generally obtained by setting $\tau=1.2$.

The effect of the two merging steps can be seen in Fig. 4.
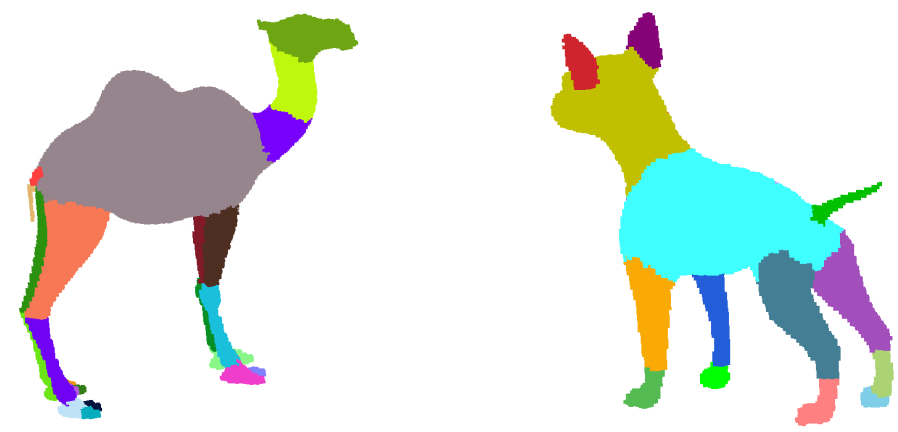

Fig. 4. Results after merging

\section{Concluding Remarks}

A method to decompose 3D objects starting from the partition of their skeleton has been presented. The elements of the skeleton partition (complex sets, single points, simple curves, segments) are then used as seeds from which to recover the various parts (kernels, bumps, simple regions, basic regions) into which the object is decomposed. In particular, simple regions may be articulated into basic regions, when 
segments are found (and are successively used as seeds) during polygonal approximation done on the corresponding simple curves. A merging process is also accomplished with the purpose of obtaining a final decomposition whose parts are in accordance with human perception and that results stable when the object is available in different poses or sizes. The suggested method with the default values of the involved thresholds has been tested on several 3D images taken from publicly available shape repositories $[15,16]$, producing generally satisfactory results.
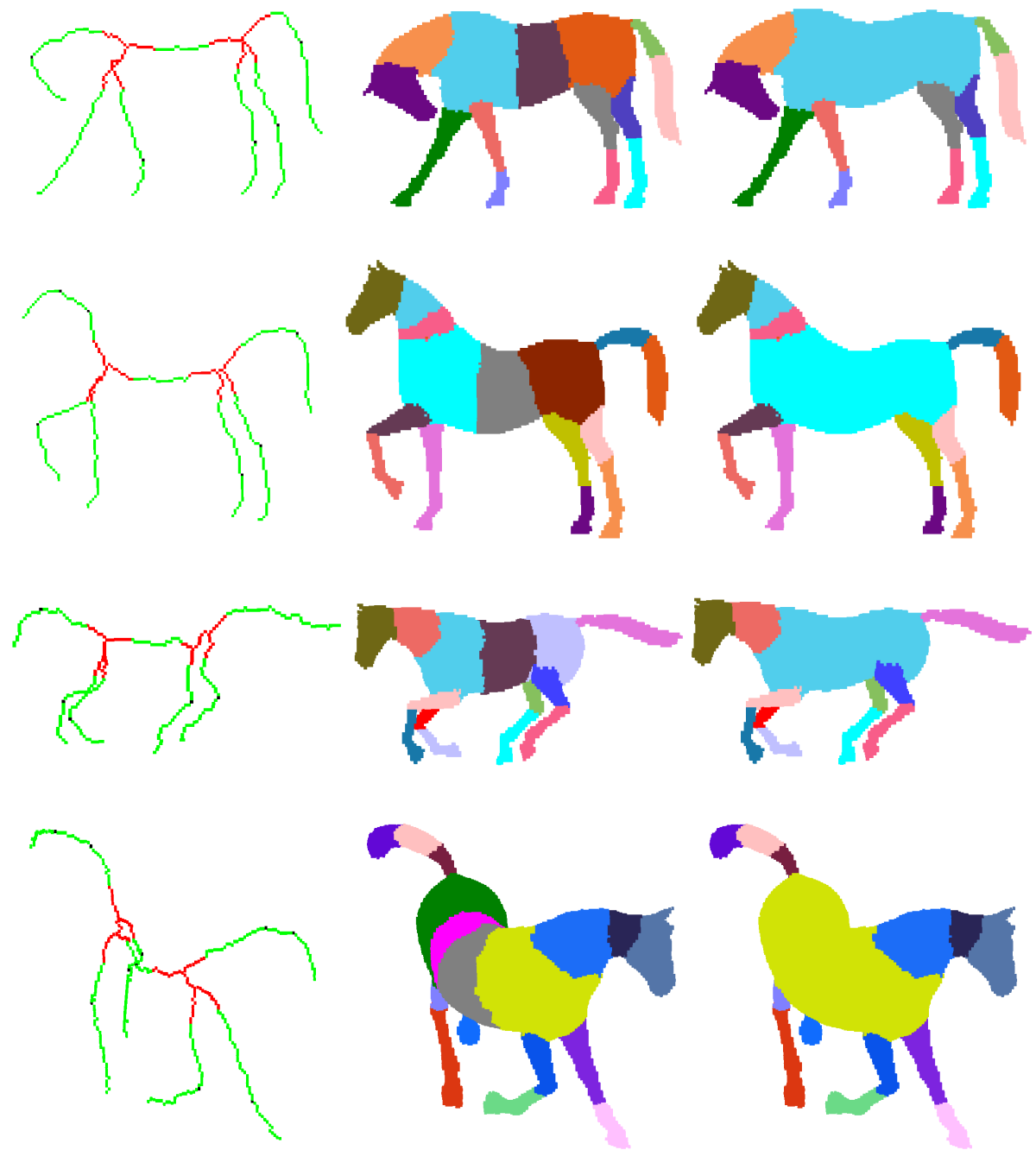

Fig. 5. Partition of the skeleton, where black voxels are the vertices of the polygonal approximation performed on the simple curves, left. Decomposition before merging, middle. Decomposition after merging, right. 
The decomposition obtained after merging can be used to identify the class to which an object belongs, in terms of the number of regions and of their spatial relationships. Moreover, by taking into account the basic regions into which simple regions may have been divided, the decomposition can be used to distinguish objects in the same class. If necessary, also the decomposition before merging can be used to derive some more information on the structure of those simple regions that have been merged to the adjacent kernels. These regions could in fact be articulated into basic regions, which account for changes in thickness or in boundary curvature.

A few examples for the object "horse" in different poses and sizes are given in Fig.5, where the partition of the skeleton, and the object decomposition before and after merging are shown. We note that the skeleton has always been partitioned into two complex sets (red voxels) and seven simple curves (green voxels). The simple curves have been divided by polygonal approximation in different subsets (the hinges, i.e., the vertices found during polygonal approximation, are shown in black). The simple region in between the two kernels has always been merged into a unique region with the kernels, even when the simple region was articulated into basic regions (Fig. 5 bottom). Thus, after merging, "horse" is always decomposed into one main body (the torso) from which six limbs (the four legs, the tail and the region including head and neck) protrude. Limbs are articulated in a different number of basic regions, reflecting the different poses of "horse". We note that bending of the torso of "horse" in Fig. 5 bottom can be pointed out by looking at the decomposition before merging. This allows us to distinguish this pose of "horse" from the remaining ones.

\section{References}

1. Hoffman, D.D., Richards, W.A.: Parts of recognition. Cognition 18, 65-96 (1984)

2. Biederman, I.: Recognition-by-components: a theory of human image understanding. Psycological Review 94(2), 115-147 (1987)

3. Singh, M., Seyranian, G.D., Hoffman, D.D.: Parsing silhouettes: the short-cut rule. Perception \& Psychophysics 61(4), 636-660 (1999)

4. Shamir, A.: A survey on mesh segmentation techniques. Computer Graphics Forum 27(6), 1539-1556 (2008)

5. Sanniti di Baja, G., Thiel, E.: (3,4)-weighted skeleton decomposition for pattern representation and description. Pattern Recognition 27, 1039-1049 (1994)

6. Cheng, Z.-Q., Li, B., Dang, G., Jin, S.-Y.: Meaningful Mesh Segmentation Guided by the 3D Short-Cut Rule. In: Chen, F., Jüttler, B. (eds.) GMP 2008. LNCS, vol. 4975, pp. 244-257. Springer, Heidelberg (2008)

7. Serino, L., Sanniti di Baja, G., Arcelli, C.: Using the Skeleton for 3D Object Decomposition. In: Heyden, A., Kahl, F. (eds.) SCIA 2011. LNCS, vol. 6688, pp. 447-456. Springer, Heidelberg (2011)

8. Blum, H.: Biological shape and visual science. J. Theor. Biol. 38, 205-287 (1973)

9. Borgefors, G.: On digital distance transform in three dimensions. CVIU 64(3), 368-376 (1996)

10. Arcelli, C., Sanniti di Baja, G., Serino, L.: Distance driven skeletonization in voxel images. IEEE Trans. PAMI 33(4), 709-720 (2011) 
11. Borgefors, G.: Digital distance transforms in 2D, 3D, and 4D. In: Chen, C.H., Wang, P.P.S. (eds.) Handbook of Pattern Recognition and Computer Vision, pp. 157-176. World Scientific, Singapore (2005)

12. Svensson, S., Sanniti di Baja, G.: Using distance transforms to decompose 3D discrete objects. IMAVIS 20, 529-540 (2002)

13. Ramer, U.: An iterative procedure for the polygonal approximation of plane curves. CGIP 1, 244-256 (1972)

14. Borgefors, G., Sanniti di Baja, G.: Analyzing non-convex 2D and 3D patterns. CVIU 63(1), 145-157 (1996)

15. AIM@SHAPE Shape Repository, http://shapes.aimatshape.net/viewmodels.php

16. Shilane, P., Min, P., Kazhdan, M., Funkhouser, T.: The Princeton Shape Benchmark, Shape Modeling International, Genova, Italy (2004) 